\title{
Le CEA et CISBIO-international créent le GIP sources HA, et révisent leur stratégie de gestion des sources scellées usagées
}

\author{
B. SEVESTRE ${ }^{1}$, B. CRABOL ${ }^{1}$, L. LAMBERTON ${ }^{1}$
}

(Manuscrit reçu le 22 Avril 2010, accepté le $1^{\text {er juin 2010) }}$

RÉSUMÉ Pour répondre à leur obligation de reprises de sources scellées devenues sans emploi ou périmées, le CEA et CISBIO international ont, en 2009, créé le groupement d'intérêt public (GIP) sources HA qui a pour objet d'assurer la récupération, le conditionnement, ainsi que l'élimination des sources radioactives scellées de haute activité (SSHA), notamment de césium-137 et de cobalt-60, qu'ils ont fabriquées et distribuées en France. Le GIP peut également reprendre les sources orphelines de même nature, ce qui inclut les sources sans détenteur autorisé et les sources sans filière de reprise ou d'élimination. Afin de récupérer l'ensemble de ces sources d'ici 2016, le GIP souhaite entrer en contact avec les utilisateurs afin d'identifier leurs besoins et leurs intentions et de planifier le retour des sources. Parallèlement, CISBIO et le CEA continuent à assurer les mêmes missions pour les autres sources dont ils ont été fournisseurs ou fabricants. Seul, ou en partenariat, le GIP, CISBIO et le CEA souhaitent optimiser cette mission de service public : (1) en développant les services connexes rendus aux utilisateurs ou aux fournisseurs actuels, et (2) en déployant une stratégie d'élimination des sources destinée à écarter le risque de saturation des capacités d'entreposage.

ABSTRACT CEA and CISBIO-international create the "GIP sources HA" and revise their strategy for the management of disused sealed sources.

According to French regulation, CEA and CISBIO, who have distributed many sealed sources in the past, have to recover all the disused sources. During 2009, CEA and CISBIO created the "groupement d'intérêt public (GIP) sources HA", with the objective of recovering and managing high-activity sealed sources (HASS), mainly cobalt-60 and caesium-137, distributed in France. CEA and CISBIO can also recover and repatriate similar sources or any other types of sealed sources distributed by them in foreign countries, when the user has no national solution, or when international organisations, such as the IAEA, identify a security issue. With the objective of optimising this mission, GIP, CEA and CISBIO are ready to cooperate with the present source suppliers who may need the use of hot cells in France, may contribute to some repatriation operations, or may recycle some batches of recovered sealed sources not yet conditioned as waste packages.

Keywords : Management / disused sources / cobalt / caesium / recycling

\footnotetext{
1 GIP sources HA, BP 18, 91192 Gif-sur-Yvette Cedex, France.
} 


\section{Pourquoi créer le GIP (groupement d'intérêt public) sources HA ?}

Le CEA et CISBIO international ont été de très importants fabricants et fournisseurs de sources scellées, encore largement utilisées dans une très large gamme d'activités aussi bien médicales, qu'industrielles ou de recherche.

Au cours de la dernière décennie, ils ont progressivement quitté ce domaine d'activité ; ainsi :

- en 1999, le CEA transférait à CERCA LEA son activité de fabrication et distribution de sources scellées étalon,

- en 2002, CISBIO transférait à CEGELEC son activité de distribution de sources industrielles de cobalt-60 (modèles COG et COGC) et césium-137 (modèles CSC et CSP),

- en 2006, CISBIO effectuait sa dernière livraison de sources scellées de haute activité.

Le CEA et CISBIO ont néanmoins conservé les activités résultant de leur obligation réglementaire de récupération et reprise des sources scellées qu'ils ont fabriquées ou distribuées en France, sur demande des utilisateurs. S'agissant des sources de haute activité de cobalt-60 et césium-137, les opérations de reprise et conditionnement sont réalisées dans les cellules THA (très haute activité) de l'INB 29, sur le centre CEA de Saclay, ancien site de production de sources et de chargement d'équipements neufs.

Parallèlement à ces évolutions, des changements importants du contexte juridique et réglementaire des relations entre le CEA et CISBIO ont été décidés et mis en œuvre :

- en 2000, CISBIO cessait d'être filiale du CEA,

- fin 2008, CISBIO (filiale d'IBA) devenait l'exploitant nucléaire de l'INB 29.

C'est dans ce contexte que le CEA et CISBIO ont pris la décision de créer le GIP sources HA, afin de fixer un nouveau cadre, de nouveaux objectifs, et une nouvelle dynamique à leurs activités communes de gestion de sources scellées usagées. Cette décision s'est concrétisée par la parution au JO du 12 juin 2009 de l'arrêté approuvant la convention constitutive du groupement.

Le GIP sources HA (dit ci-après le GIP) a pour objet d'assurer la récupération, le conditionnement, ainsi que l'élimination des sources radioactives scellées de haute activité (SSHA), notamment de césium-137 et de cobalt-60 fabriquées et distribuées en France par le CEA et par CISBIO, ainsi que les sources orphelines de même nature.

Le GIP exerce ses missions en recherchant toute opportunité d'optimisation économique. La création du GIP est complétée côté CEA par une «mission 
sources », rattachée à la direction en charge des assainissements et démantèlements du CEA, et côté CISBIO par une « activité sources ». La mission sources du CEA a en charge le pilotage des activités de gestion de toutes les sources scellées usagées distribuées par le CEA, ou utilisées par le CEA, ou dont la gestion est en partie confiée au CEA par l'ANDRA ; elle assure aussi les relations entre le CEA et le GIP. L'activité sources de CISBIO a en charge les prestations de CISBIO dans le cadre du GIP et l'ensemble des autres activités de CISBIO touchant aux sources scellées : maintenance d'équipements ou de sources, prestations utilisant les cellules THA de l'INB 29, reprise des sources distribuées par CISBIO et ne relevant pas de l'objet du GIP. Dans la suite de l'article, nous aborderons donc l'ensemble des activités du CEA, de CISBIO et du GIP concernant la gestion des sources scellées usagées, sans nous limiter à celles relevant strictement de l'objet du GIP sources HA.

\section{Améliorer et développer les services rendus par le GIP sources HA, le CEA et CISBIO aux utilisateurs de sources scellées (Fig. 1)}

\subsection{Récupération et reprise des sources scellées sans emploi}

Conformément à leurs obligations réglementaires, le CEA et CISBIO organisent la collecte et la reprise des sources scellées usagées qu'ils ont fabriquées ou distribuées. Ceci est fait sur demande des utilisateurs, dès lors qu'ils n'en ont plus l'usage ou qu'elles ont atteint leur limite réglementaire d'utilisation. Les tarifs et les conditions générales de vente du GIP sont publics, et peuvent être communiqués sur demande, afin de permettre aux utilisateurs de planifier les budgets nécessaires.

\section{Planification des retours}

Dans le cas des sources scellées de haute activité, le GIP va mettre en place une planification des retours, afin de permettre une optimisation de ses capacités et de ses opérations. En effet, la maîtrise des coûts générés par la gestion des sources scellées usagées est essentielle, pour que le tarif de reprise reste acceptable par les utilisateurs. Or la disponibilité et le taux d'utilisation des cellules THA de l'INB 29 sont des éléments directeurs essentiels pour une partie de ces coûts : le CEA et CISBIO ont fixé au GIP l'objectif de minimiser la durée d'utilisation des cellules THA et de maximiser leur taux d'utilisation pendant cette durée. Outre une nécessité économique, cet objectif est également rendu nécessaire par l'incompatibilité des opérations de reprise et conditionnement de sources scellées de haute activité avec les opérations d'assainissement et démantèlement des cellules ayant servi à la fabrication des sources. Pour mettre en place cette planification, le GIP cherche à entrer en contact avec tous les utilisateurs ou détenteurs de sources scellées de 
Obligations règlementaires :

Autres services :

GIP sources HA :

Récupération et reprise des

SSHA cobalt et césium

distribuées en France par

CEA et CISBIO

\section{CISBIO :}

Récupération et reprise des

autres sources distribuées

en France par CISBIO
CEA :

Récupération et reprise des

autres sources distribuées

en France par le CEA

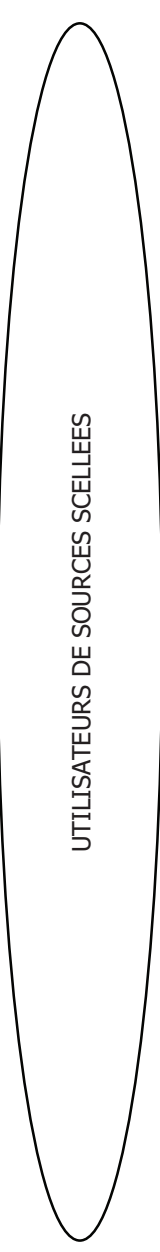

Récupération et reprise de source orphelines en France sour

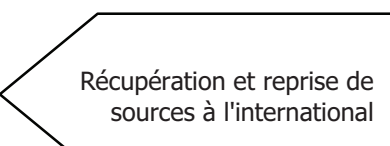
sources à l'international

CEA et CISBIO

contribution aux demandes

de prolongation d'utilisation

de sources scellées, en

tant que fournisseurs ou

fabricants

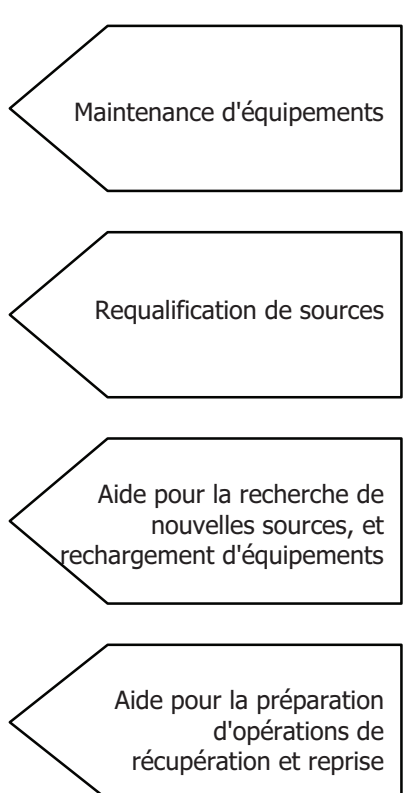

Selon le cas, les services indiqués ci-dessus sont rendus par le GIP, CISBIO, le CEA ou par un prestataire ou partenaire.

Figure 1 - Services rendus par le GIP sources HA, le CEA ou CISBIO.

Services proposed by the GIP sources HA, by CEA and by CISBIO. 
haute activité, de cobalt-60 ou césium-137 distribuées par le CEA ou CISBIO ou orphelines, avec l'objectif de les récupérer avant 2016, soit dix ans après les dernières fournitures.

\section{Récupération et reprise des sources orphelines}

L'objet du GIP inclut la reprise des sources dites orphelines : cette mission de service public va au-delà des obligations réglementaires du CEA et de CISBIO, et a pour objectif d'offrir une solution concrète pour la récupération des sources de même nature (sources scellées de haute activité), mais qui répondraient à l'une des situations suivantes :

- soit elles sont retrouvées sans détenteur autorisé,

- soit le détenteur (utilisateur, ou fournisseur ayant repris une source) ne dispose d'aucune filière de reprise ou d'élimination, ce qui peut correspondre à des situations très variées : fournisseur ou fabricant non identifié, fournisseur ou fabricant ayant disparu, absence d'engagement de reprise par le fournisseur ou le fabricant, incapacité d' un fournisseur ou fabricant d'honorer son engagement de reprise du fait d'une évolution de la loi ou de la réglementation dans le pays d'origine, fournisseur ne disposant pas de filière d'élimination pour les sources qu'il a reprises... Les capacités du GIP à satisfaire ce type de besoin sont immédiates en ce qui concerne les sources de cobalt-60 et césium-137; elles nécessiteront un examen au cas par cas pour les autres radioéléments.

Récupération et reprise de sources scellées de très haute activité à l'international

Par ailleurs, le CEA, CISBIO et l'IRSN examinent avec l'AIEA et les autorités françaises concernées les modalités qui permettraient la sécurisation de sources scellées de très haute activité (notamment sources des catégories 1 et 2 selon les standards de l'AIEA) ou d'équipements contenant de telles sources, de fourniture française, dans des pays ne disposant pas de l'expertise, des installations ou des ressources nécessaires. Ces opérations de sécurisation seraient selon les cas réalisées dans le pays détenant les sources, ou pourraient nécessiter un rapatriement. Le CEA et CISBIO assurent également ponctuellement des reprises de sources qu'ils ont distribuées à l'international, quand l'utilisateur ne dispose d'aucune autre solution de gestion.

\subsection{Aide à la préparation d'opérations de reprise, et optimisation de la collecte}

Caractérisation de lots mixtes de sources et de déchets

Certains utilisateurs ont accumulé au cours du temps des lots de sources sans emploi d'origines diverses, parfois incomplètement caractérisées, pouvant comporter 
des sources scellées, ainsi que des sources non scellées, voire des objets radioactifs n'ayant pas le statut de source et relevant donc d'une filière de déchets radioactifs. Afin d'offrir une solution concrète à ce type de situations, le CEA souhaite saisir l'opportunité de la complémentarité des diverses installations existantes sur le site du CEA de Saclay pour mettre en place, en collaboration avec l'ANDRA, un processus en deux phases, qui sera proposé à ces détenteurs :

- phase de préparation : cette phase a pour objectif de caractériser les sources et objets à éliminer et préparer leur récupération (transport et orientation au cas par cas vers une filière déchets ou une filière sources scellées) ;

- phase de collecte : sur la base d'un document technique issu de la phase de préparation, le CEA et l'ANDRA organiseront une collecte commune des sources et déchets à récupérer, et leur reprise par l'installation la mieux adaptée du site de Saclay : installation ANDRA de collecte des déchets radioactifs des petits producteurs, installation CEA de collecte des sources de faible activité au sein de l'INB 49. Le cas échéant, le CEA pourra réorienter certaines sources vers les installations de l'INB 29 dédiées aux activités du GIP, pour des besoins d'optimisation.

\section{Caractérisation de sources scellées irradiantes}

Dans certains cas, la caractérisation (identification et contrôle de non contamination) d'une source scellée de haute activité peut nécessiter une intervention en milieu trop irradiant pour une intervention manuelle, alors que le détenteur ne dispose pas ou ne dispose plus des moyens permettant de réaliser ces gestes dans des conditions satisfaisantes de radioprotection : le détenteur ou le GIP peuvent dans ces cas faire appel aux moyens d'intervention par robots de l'IRSN/SIAR (la faisabilité d'une intervention de ce type nécessite un examen au cas par cas en fonction de caractéristiques de la sources, de son contenant, et des locaux).

\section{Capacités du SAV de CISBIO et capacités de transport}

Par ailleurs, la récupération de certaines sources scellées de très haute activité est indissociable de celle de l'équipement les contenant et nécessite une préparation, une intervention sur site et un transport spécifiquement étudiés et organisés : le GIP sources HA dispose pour ces opérations du savoir faire des équipes de service après vente (SAV) de CISBIO et d'un parc d'emballages de transport dédiés au GIP, complété si nécessaire par des locations d'emballages. Afin d'améliorer leurs capacités de récupération d'équipements et de sources, le CEA et CISBIO ont également développé en commun une sur-coque de transport, dont la fabrication et l'agrément sont en cours ; cette sur-coque a vocation à pouvoir être utilisée en complément de l'équipement, quand celui-ci a perdu son agrément d'emballage de transport. Selon les types d'équipement ou de sources, des compléments d'agréments ou des arrangements 
spéciaux pourront s'avérer nécessaires : ces opérations doivent donc être largement anticipées et planifiées, pour permettre leur préparation et leur réalisation dans des délais compatibles avec les besoins des utilisateurs.

\subsection{Aide à la pérennisation d'équipements et/ou de sources}

Les contacts pris par le GIP ou par le CEA auprès de certains utilisateurs ont mis en évidence dans de nombreux cas un besoin de pérennisation de sources ou d'équipements. Ces situations peuvent correspondre à des besoins très variés : simple besoin de maintenance d'équipement sur site, demande de contribution à un dossier de demande de prolongation d'utilisation de source pouvant inclure une requalification de sources en cellules THA, demande d'aide à la recherche d'un fournisseur capable de fournir une source adaptée aux besoins de l'utilisateur. Du point de vue de l'utilisateur, ces services complémentaires sont indissociables des opérations de récupération et reprise des sources scellées usagées. Ces opérations de pérennisation sortent de l'objet du GIP et seront chaque fois que possible réalisées dans le cadre de collaborations industrielles, ou à défaut par le CEA ou CISBIO.

Opérations incluant la fourniture de nouvelles sources

Le GIP, CISBIO et le CEA s'attachent pour cette raison à développer leurs capacités de service. Ceci nécessite un dialogue ouvert avec les actuels fournisseurs de sources, car ni le CEA, ni CISBIO, ni le GIP n'ont vocation à fournir des sources neuves ou recyclées. Pour certains modèles n'ayant pas d'équivalent, CISBIO envisage de céder à d'autres fabricants un droit d'usage du design d'origine, afin de répondre aux besoins de certains utilisateurs.

Une opération de ce type peut typiquement nécessiter successivement :

- une opération sur site par le SAV de CISBIO suivie d'un transport,

- le déchargement de l'équipement dans les cellules THA de l'INB 29 pour reprise des sources usagées par le GIP,

- la maintenance de l'équipement par CISBIO, suivie de son rechargement en cellules avec des sources neuves dont le fournisseur a été choisi par l'utilisateur : c'est ce fournisseur qui sera à l'avenir le porteur de l'obligation réglementaire de reprise,

- transport vers le site de l'utilisateur et opérations de SAV sur site pour remise en service de l'équipement.

Prolongation d'utilisation et requalification des sources scellées

La poursuite de l'utilisation de sources scellées au-delà de dix ans constitue également un besoin fréquent et récurrent, dont les modalités ont été récemment explicitées, par la publication au JO de l'arrêté du 23 octobre 2009. Le CEA et CISBIO 
s'attacheront à répondre au mieux aux besoins des utilisateurs pour la mise en œuvre de ces nouvelles dispositions réglementaires, tout en veillant à ne pas obérer l'objectif déjà cité de limiter à quelques années seulement la durée d'utilisation des cellules THA de l'INB 29, particulièrement bien adaptées à la requalification des sources scellées de haute activité cobalt-60 ou césium-137. Notamment, ces cellules permettent de réaliser un test d'étanchéité à l'hélium ou par bullage, et un examen visuel d'absence de corrosion ou d'agression mécanique de la source.

\section{Vers une nouvelle stratégie du CEA pour l'élimination des sources scellées usagées}

Les fournisseurs de sources doivent, par obligation réglementaire, disposer des capacités d'entreposage nécessaires à leur reprise effective. Mais cette obligation soulève des difficultés concrètes qui risqueraient de conduire à une impossibilité de reprise de certaines sources, si des voies d'élimination des sources reprises ne sont pas rapidement ouvertes.

\subsection{Critères d'acceptation des sources en stockage}

Dans la cadre du plan national de gestion des matières et déchets radioactifs, élaboré au titre de la loi du 29 juin 2006 sur la gestion des déchets radioactifs, l'ANDRA a établi fin 2008 et rendu publique début 2009 une étude sur la gestion durable des sources scellées usagées. Cette étude complète la spécification diffusée en 2006 pour l'acceptation de colis de déchets contenant des sources scellées au CSFMA et propose des critères d'acceptation des sources scellées pour l'ensemble des stockages de déchets existants ou envisagés, dès lors que la décision a été prise de les gérer en tant que déchets. Les avis de l'IRSN et de l'ASN sur cette étude ont également été rendus publics en cours d'année, et la gestion des sources scellées usagées est abordée dans la nouvelle édition du PNGMDR.

Les critères proposés par l'ANDRA, (et qui devront encore dans certains cas être précisés, confirmés ou ajustés), peuvent se résumer ainsi (il s'agit, tel qu'ils sont reformulés ici par les auteurs, de règles générales qui pourront comporter des exceptions justifiées) :

- les producteurs devront fabriquer et caractériser des colis primaires de sources scellées, en évitant les mélanges de sources et de déchets,

- le procédé de fabrication des colis devra éviter de détériorer les sources, afin de ne pas perdre le bénéfice de confinement apporté par la technologie des sources scellées,

- pour les centres de stockage de surface ou à faible profondeur, une limite d'activité par source (LAS) sera définie pour tous les radioéléments de période 
radioactive supérieure à celle du cobalt-60. Ce critère qui tient compte de la géométrie de chaque source, permettra de maîtriser les conséquences d'une éventuelle manipulation de la source à nu, à la suite d'une intrusion dans le stockage qui surviendrait au-delà de sa période d'exploitation et de surveillance (il en résulte que la période radioactive sera un critère important d'orientation des sources vers les stockages autres que le stockage géologique),

- pour l'ensemble des stockages, les colis de sources devront également respecter les critères applicables aux autres colis de déchets (sont notamment à considérer selon les stockages des critères sur les dimensions et la masse des colis, sur l'activité massique, la radioprotection et la puissance thermique des colis, sur l'état physique, ainsi que la toxicité, et la quantification de certains éléments chimiques des sources, équipements ou protections inclus dans les colis).

\subsection{Stratégie de référence du CEA pour l'élimination des sources scellées usagées par mise en colis de déchets}

Au moment de la parution de l'étude ANDRA déjà citée, le CEA rencontrait des difficultés pour assurer le fonctionnement pérenne de ses filières de reprise, en raison d'un risque de saturation de ses capacités d'entreposage des sources scellées. Les données nouvelles contenues dans cette étude ont donc été considérées comme une opportunité : le CEA a engagé en 2009 une refonte de sa stratégie, et a défini une solution de référence pour l'élimination des sources en colis de déchets, qui seront entreposés puis orientés vers les centres de stockage ANDRA (d'autres voies susceptibles de compléter cette stratégie sont explorées au paragraphe 3.3 ciaprès). Cette stratégie fera l'objet en 2010 et 2011 dans le cadre global d'un examen de la gestion des déchets radioactifs par le CEA, d'une instruction par l'IRSN et sera soumise à l'avis de l'ASN et du DSND. Sa mise en ouvre nécessitera un dialogue approfondi avec l'ANDRA, pour accord sur la définition des colis de déchets sources. Pour les sources relevant de leurs responsabilités, l'ANDRA et d'autres détenteurs de stocks de sources scellées usagées pourront également être des utilisateurs de ces filières CEA; le CEA est en effet ouvert à des collaborations sur le développement de ses filières, afin d'en optimiser l'usage et le coût.

Outre les données d'entrée provenant de l'ANDRA et des avis IRSN et ASN déjà cités, cette stratégie de référence prend en compte :

- les types et quantités de sources CEA à gérer (en tenant compte du partenariat avec CISBIO dans le cadre du GIP, et des prestations demandées au CEA par l'ANDRA pour certaines sources, notamment les sources et objets au radium),

- les contraintes d'irradiation au moment du conditionnement des sources, et après conditionnement,

- la nécessité économique d'utiliser chaque fois que possible des filières déchets existantes ou prévues, que ce soit pour l'entreposage ou pour la mise en stockage, 
- la nécessité économique d'utiliser chaque fois que possible des emballages de transport existant ou prévus,

- les contraintes relatives à la situation actuelle (localisation, conditionnements déjà réalisés) des sources scellées déjà reprises dans le passé (ces stocks constituent plus de la moitié de l'inventaire des sources à gérer par le CEA et le GIP).

Cette stratégie de référence est synthétisée ci-après et dans la figure 2. Sa mise en œuvre étant en cours d'étude et compte tenu des avis attendus de l'IRSN, puis de l'ASN et du DSND, certaines de ses modalités sont encore susceptibles d'évolutions ; de plus, certaines sources sont compatibles avec plusieurs filières, ce qui fournira lors de la mise en œuvre une opportunité d'optimisation des flux entre filières.

\section{Élimination des lots de sources scellées de forte puissance thermique}

Les stocks repris ou à reprendre à court terme représentent dans certains cas une forte puissance thermique sous un faible volume, ce qui nécessite des filières d'élimination spécifiques. Pour le cobalt-60, une trentaine d'emballages de transport seraient utilisés comme colis primaires pour l'envoi de sources au CSFMA : l'ensemble représente environ 3500 watts (valeur cumulée des colis à la date de leur envoi en stockage, prévue en dix ans à partir de 2014). Pour les radioéléments à vie plus longue et notamment le césium-137, environ 7 colis de déchets technologiques au format des colis de verre du CEA seraient réalisés à l'occasion d'une campagne unique vers 2015/2016, puis entreposés dans les mêmes conditions que les colis de verre du CEA (de même géométrie) puis envoyés en stockage des déchets à haute activité et vie longue (HAVL) : l'ensemble représente environ 1000 watts à la date d'envoi en stockage, prévue vers 2030.

Ces deux filières permettront notamment l'élimination des stocks et des flux attendus à court terme de sources hautement irradiantes en gamma ; pour les flux résiduels ultérieurs, d'autres filières sont définies ci-après.

\section{Élimination des sources scellées à vie courte}

Les plaques et disques étalons d'usage médical en cobalt-57 et germanium-69 ont une période radioactive très courte, inférieure à un an, et un volume important ; ces sources seraient éliminées en environ trois caissons d'un $\mathrm{m}^{3}$ en tant que déchets de très faible activité, destinés au CSTFA.

Les sources scellées de cobalt-60 de faible activité seraient éliminées en une dizaine de colis cimentés, destinés au CSFMA.

Les sources scellées de tritium seraient éliminées (compte tenu de leur très faible volume) en mélange avec les déchets traités, dont la gestion fait l'objet de travaux spécifiques dans le cadre du PNGMDR. 
Elimination de sources scellées en tant que déchets.
Autres voies explorées par le CEA.
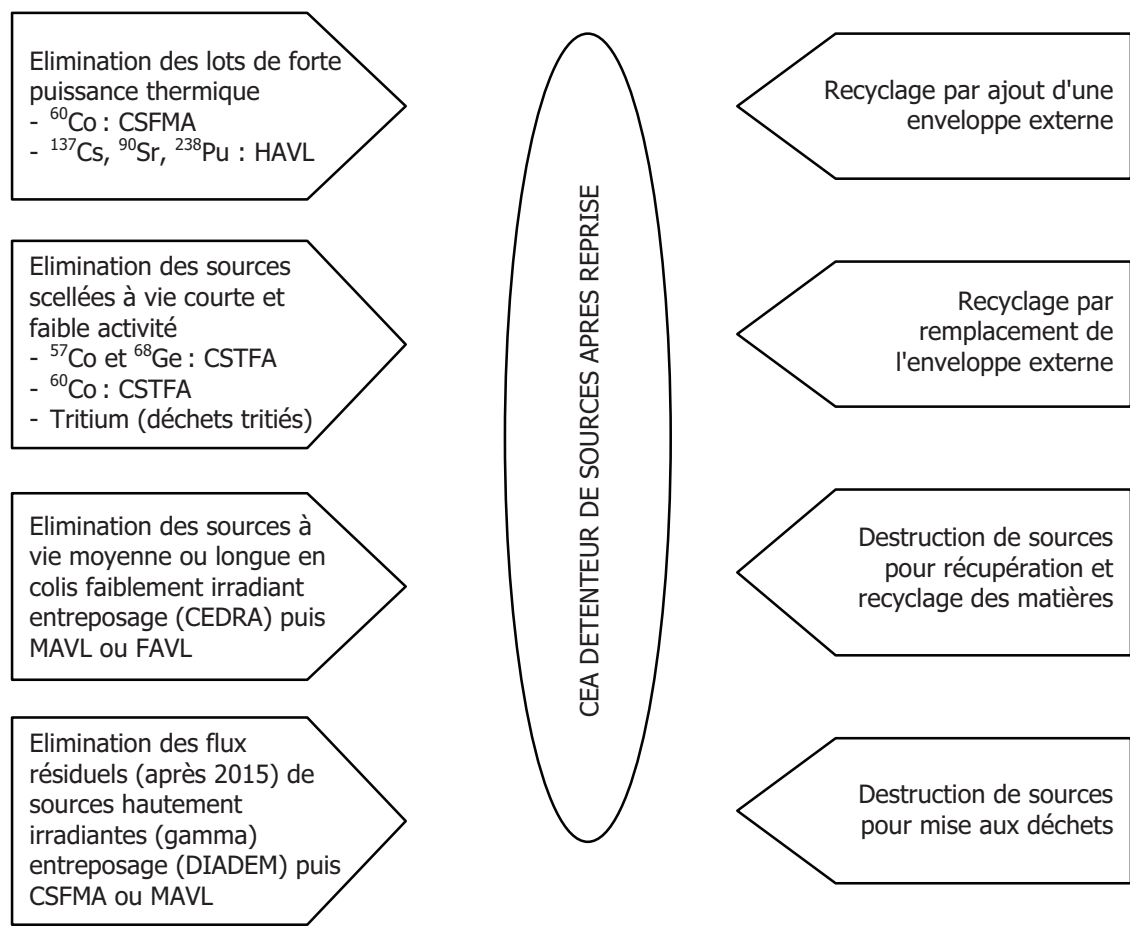

Le CEA examinera toute opportunité de collaboration avec d'autres détenteurs de sources, ou avec des prestataires en vue de l'optimisation de ses filières d'élimination.

Figure 2 - Stratégie d'élimination des sources scéllées usagées.

Strategy for the elimination of disused sealed sources.

\section{Élimination des sources à vie moyenne ou longue en colis faiblement irradiants}

Un très grand nombre de sources (notamment les sources utilisées dans les détecteurs ioniques d'incendies, les sources de haute activité alpha ou neutroniques, et les sources de faible ou moyenne activité de tous radioéléments) pourraient être conditionnées en colis cimentés faiblement irradiants, puis entreposés dans l'installation CEDRA de CEA à Cadarache, avant envoi en stockage. Chaque colis serait orienté, selon son contenu, soit vers le stockage MAVL, soit vers le stockage FAVL. Le nombre de colis de sources à produire dans cette filière est estimé 
à 40, venant s'ajouter à un même nombre de colis dits « blocs sources » ayant été repris du Centre Manche de l'ANDRA avant fermeture de celui-ci.

Élimination des flux résiduels de sources hautement irradiantes (gamma)

La plupart des stocks de sources hautement irradiantes en gamma seraient éliminées dans le cadre des lots de sources scellées de forte puissance thermique déjà présentés ci-dessus ; les flux résiduels seraient conditionnés pour entreposage de décroissance dans une installation de gestion des déchets irradiants prévue au CEA/Marcoule pour les déchets issus du démantèlement des réacteurs du CEA : projet DIADEM.

\subsection{Autres voies explorées par le CEA}

Outre l'élimination en colis de déchets sources, deux autres voies ont été dans le passé et seront à nouveau explorées par le CEA, en complément de la stratégie de référence : d'une part la voie du recyclage des sources scellées et d'autre part la voie de la destruction des sources.

Recyclage des sources

Au plan des principes, la voie du recyclage est préférable chaque fois qu'elle est techniquement et économiquement réalisable. Trois procédés sont à envisager :

- la fabrication d'une source en encapsulant une ou plusieurs sources à recycler dans une nouvelle enveloppe (ou double enveloppe) de plus grandes dimensions,

- la fabrication d'une source de même dimension en remplaçant l'enveloppe externe de la source usagée,

- la récupération des matières premières pour remplacement complet des enveloppes scellées.

Le CEA et CISBIO n'ont pas vocation à réaliser ces opérations, mais tiennent à la disposition des fabricants de sources la liste des sources en attente de conditionnement en déchets dont ils disposent, afin de permettre des décisions au cas par cas sur les opportunités techniques et économiques de recyclage.

\section{Élimination des sources par destruction}

Pour certaines technologies de sources d'activité moyenne ou faible, notamment celles dont la compatibilité physique ou chimique avec les filières d'élimination définies ci-dessus soulèveraient des difficultés, la voie de la destruction complète pourra être examinée. Les matériaux issus de la destruction seraient alors traités selon une filière ordinaire de déchets radioactifs adaptée à leur nature physicochimique. 


\section{Conclusions}

La maîtrise de la gestion des sources scellées usagées représente des enjeux importants de sécurité, de sûreté, de radioprotection, et doit de plus être optimisée économiquement afin que son coût reste acceptable, tant pour les utilisateurs de sources que pour les fournisseurs, parfois détenteurs de stocks importants de sources scellées usagées. Le GIP sources HA, le CEA et CISBIO souhaitent développer les contacts avec les utilisateurs et fournisseurs de sources, et ceci le plus en amont possible afin de répondre de manière planifiée et optimisée à l'ensemble des besoins constatés, en tenant compte des délais nécessaires aux investissements, autorisations, ouvertures et aménagements de filières. 